\title{
Imaging and Spectroscopy of Interfaces and Surfaces with Advanced Electron Microscopy
}

\author{
Y. Zhu, ${ }^{*}$ H. Inada, ${ }^{* *}$ D. Su, ${ }^{*}$ L. Wu, ${ }^{*}$ J. Ciston, ${ }^{*}$ Ch. Jooss, ${ }^{* * *}$ J. Norpoth, ${ }^{* * *}$ and J. Wall* \\ * Brookhaven National Laboratory, Upton, NY 11973 USA \\ ** Hitachi High Technologies Corp., Ibaraki Japan \\ *** University of Gottingen, Gottingen, Germany
}

Of prime importance in the study of materials' interfaces is the use of Transmission Electron Microscopy (TEM) and Scanning Transmission Electron Microscopy (STEM) to examine the interfacial crystal structure, strain distribution, dopant profile and bonding state to understand the interfacial behavior. Traditionally, TEM has been the method of choice. With the development and implementation of aberration correction nanoprobe high-angle-annular-dark field (HAADF) imaging and energy loss spectroscopy in STEM become increasing popular with extraordinary ability to quantitatively study interface structure at atomic scale.

At Brookhaven, we have three aberration corrected electron microscopes, i.e., the Hitachi HD2700C STEM, the FEI Titan 80-300 E-TEM, and the JEOL 2200MCO [1]. The Hitachi STEM is equipped with a cold-field-emission electron source, a probe corrector and a high-resolution energy-loss spectrometer. The Titan E-TEM has an imaging corrector and an environment cell that allows us to inject various gases up to $20 \mathrm{mbar}$ for in-situ chemical reaction experiment. The JEOL 2200MCO consists of an in-column omega filter, two aberration correctors and a monochromator. We have been using these instruments to study interfaces and surfaces of advanced materials.

Figure 1 shows an example of studying $\mathrm{YBa}_{2} \mathrm{Cu}_{3} \mathrm{O}_{7} / \mathrm{Pr}_{0.5} \mathrm{Ca}_{0.5} \mathrm{MnO}_{3}$ interfaces with simultaneous acquisition of HAADF-STEM and energy-loss spectroscopy imaging, utilizing the Ca-L, Mn-L, Ba$\mathrm{M}$ and $\mathrm{Pr}-\mathrm{M} / \mathrm{Cu}-\mathrm{L}$ edges, to reveal interfacial atomic arrangement, chemical composition and electronic structure of the strongly correlated functional oxide. With a $0.1 \mathrm{~nm}$ probe of the Hitachi HD2700C we were able to determine the termination layer of the interface as well as the valence state and interfacial diffusion of the transition-metal elements in the material that exhibits intriguing physical properties. Figure 2 gives an example of atomic image of the surface structure of $\mathrm{YBa}_{2} \mathrm{Cu}_{3} \mathrm{O}_{7-\mathrm{x}}$ superconductor recorded using secondary electrons with unprecedented resolution. The corresponding HAADF-STEM image was simultaneously acquired as a reference for quantitative analysis [2]. The ability to image surface and bulk at atomic resolution is unparalleled in comparison to any known imaging techniques and has the potential to revolutionize the field of microscopy and imaging. The method we developed can bring material research to a new dimension [3].

\section{References}

[1] Y. Zhu, and J. Wall, "Aberration-corrected electron microscopes at Brookhaven National Laboratory", Book chapter in Aberration-corrected Electron Microscopy, P.W. Hawkes, Ed., Elsevier/Academic Press, p.481-523 (2008).

[2] Y. Zhu, H. Inada, K. Nakamura, and J. Wall, "Imaging single atoms using secondary electrons with an aberration-corrected electron microscope", Nature Materials, 8808 (2009).

[3] Work supported by the U.S. DOE, Office of Basic Energy Science, under Contracts No. DEAC02-98CH10886. 

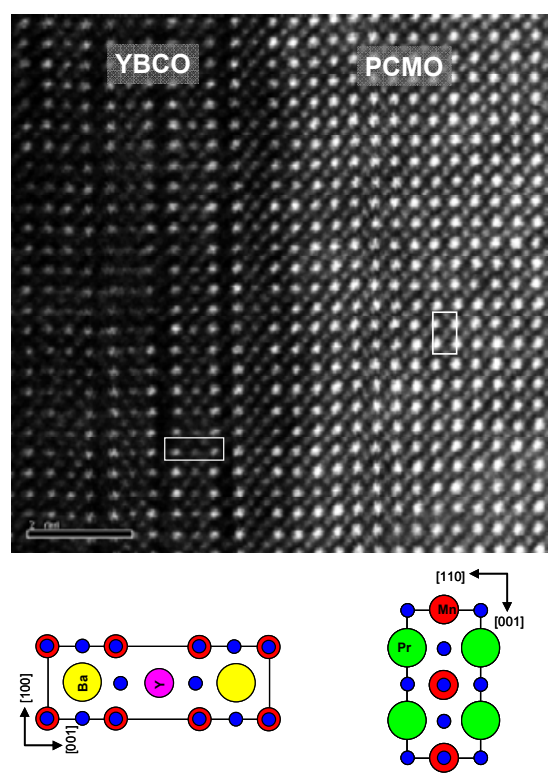

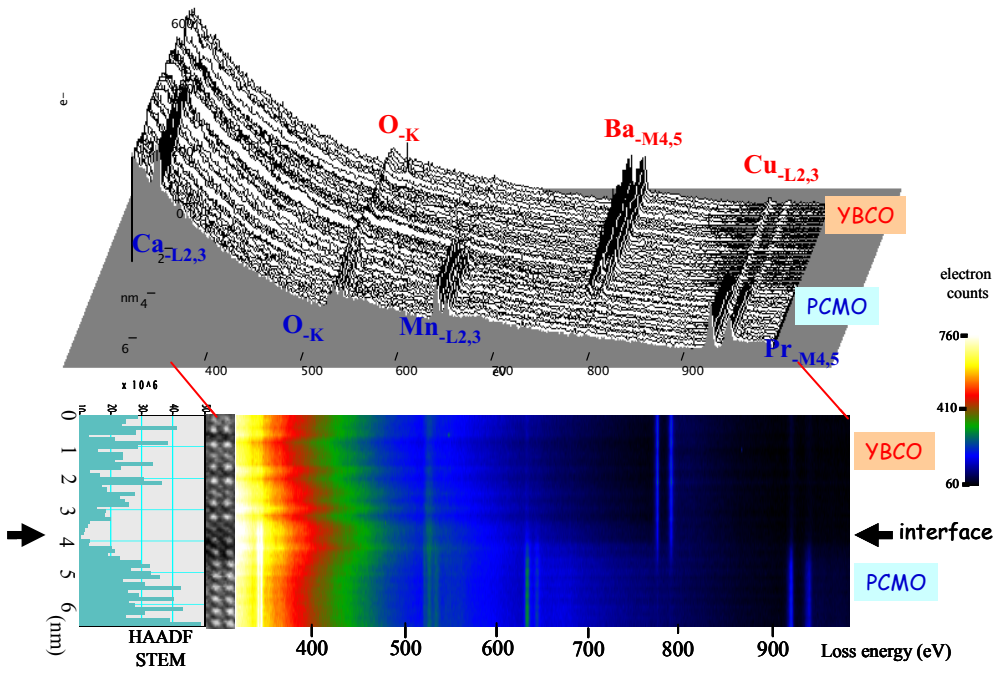

Fig.1 Interface of $\mathrm{YBa}_{2} \mathrm{Cu}_{3} \mathrm{O}_{7} / \mathrm{Pr}_{0.5} \mathrm{Ca}_{0.5} \mathrm{MnO}_{3}$. Simultaneous acquisition of HAADF-STEM image (left) and energy-loss spectroscopy imaging (right), containing $\mathrm{Ca}-\mathrm{L}, \mathrm{Mn}-\mathrm{L}, \mathrm{Ba}-\mathrm{M}$ and $\operatorname{Pr}-\mathrm{M} / \mathrm{Cu}-\mathrm{L}$ edges, to understand interfacial atomic arrangement, chemical composition and electronic structure of the strongly correlated oxide. The termination layer and sharpness of the interface as well as the valence state and interfacial diffusion of the cations are determined.
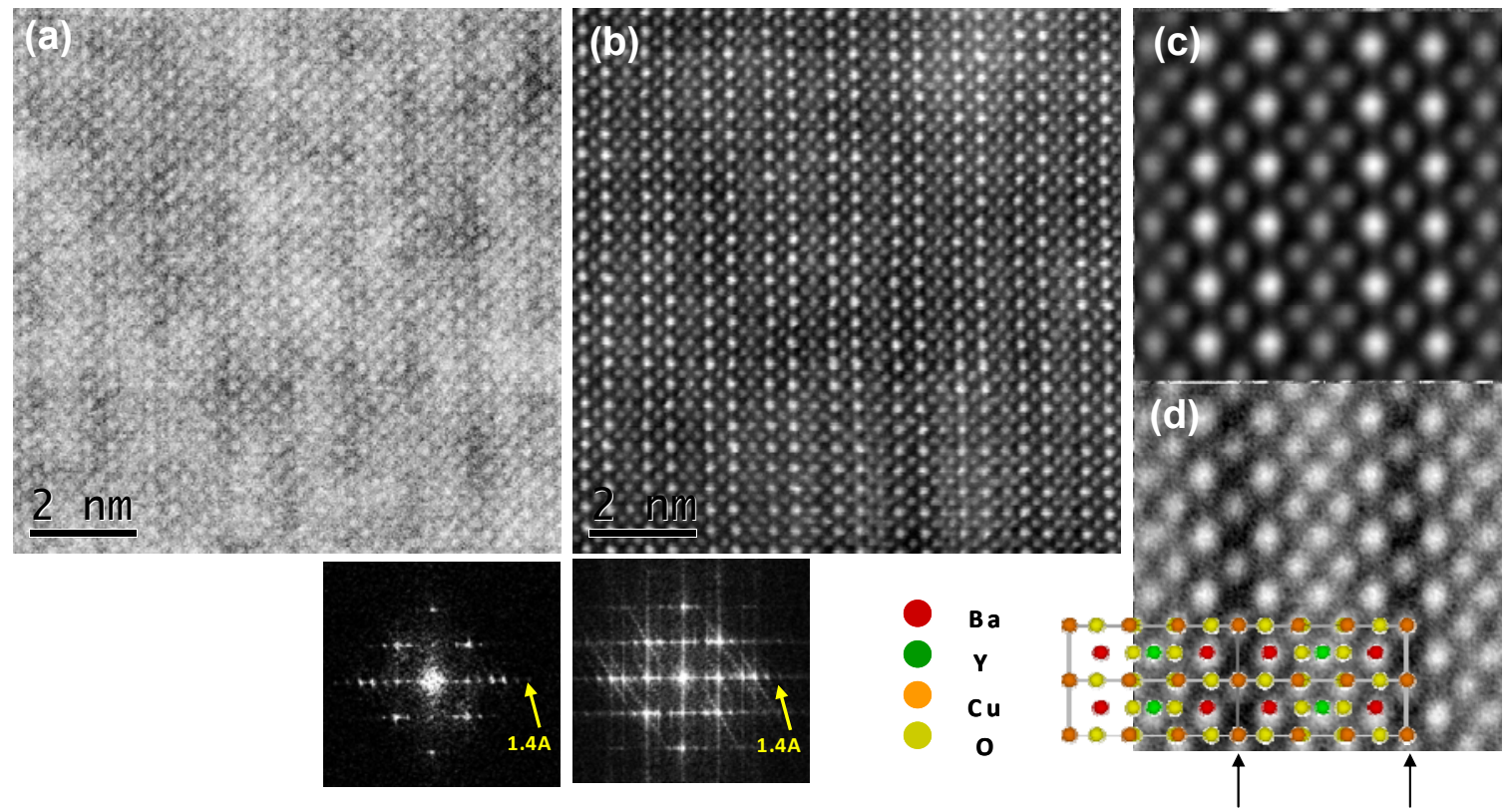

Fig.2 Surface of $\mathrm{YBa}_{2} \mathrm{Cu}_{3} \mathrm{O}_{7-\mathrm{x}}$. Simultaneous acquisition of the SEM image using secondary electrons (a) and HAADF-STEM image (b) viewing along the [010] direction. The corresponding FFT are shown below. EELS measurement suggests the thickness of the area is about $52 \mathrm{~nm}(\mathrm{t} / \lambda=0.45)$. (c- $\mathrm{d})$ A magnified averaged STEM (c) and SEM (d) image of the average of 55 unit-cells. Superimposed is the structure model (2-unit-cell in the [010] projection). Note the dark vertical lines in the SEM image (arrows) that are not evident in the STEM image. These locations correspond to the $\mathrm{CuO}$ chains with no oxygen atoms in between. We expect better contrast of light atoms on a cleaved surface since the sample we show here was thinned by ion-milling and its surface condition was not ideal. 